\title{
Analysis of Multifrequency Impedance of Biologic Active Points Using a Dry Electrode System
}

\author{
Min Soo Kim, PhD, , Young-Chang Cho, PhD, ${ }^{2}$ Suk-Tae Seo, PhD, \\ Chang-Sick Son, $\mathrm{PhD}^{3}$, and Yoon-Nyun Kim, MD, $\mathrm{PhD}^{1,4}$
}

\begin{abstract}
Objectives: A system is being developed for measurement of biologic active points (BAPs) in humans using a modified dry electrode. The BAPs measuring system analyzed the electrical characteristics and searched for the position of BAPs using modified dry electrodes.

Subjects: Skin electrical resistance at BAPs and non-BAPs was examined with a modified electrode system for healthy male subjects (ages 21-40). Four (4) acupuncture points of PC-4, PC-5, PC-6, and PC-7 on the left arms were chosen for BAPs. Bio-impedance was then conducted for BAPs and non-BAPS using a lock-in amplifier with a frequency range of $1 \mathrm{~Hz}-1 \mathrm{kHz}$.

Results: Resistances of four BAPs were found to decrease to about $29 \%-59 \%$ of non-BAPs and reactance of BAPs was found to decrease to about $23 \%-41 \%$ of non-BAPs. The difference in electrical impedance between BAPs and non-BAPs was easily recognized since the average value of BAPs was measured at lower values than that of nonBAPs. Through these experiments, BAPs could be distinguished from non-BAPs based on electrical impedance. In addition, the electrical impedance model used—the electrical BAPs model—appears to be better suited for skin.

Conclusions: The proposed BAPs electrical model of skin can be adapted for interpretation of changes in the impedance characterization of skin. This system would be used for various skin diagnoses due to the simplicity and reliability of bio-impedance analysis.
\end{abstract}

\section{Introduction}

A CUPUNCTURE POINTS OR BIOLOGIC ACTIVE POINTS (BAPS) have been used for thousands of years for treatment of a variety of ailments, including cardiovascular diseases. According to Traditional Chinese Medicine, there exists a relationship between organs and BAPs in the human body. ${ }^{1,2}$ BAPs are anatomically and topographically defined as functional entities, often subjectively identified as tender points, and are frequently described as having characteristic electrical properties. ${ }^{3}$ BAPs may be characterized by increased conductance, reduced impedance/resistance, increased capacitance, and elevated electrical potential. ${ }^{4}$ Many commercial electrodiagnostic devices employ these properties for precise location of acupuncture points and for various clinical applications.

Bioelectrical impedance analysis has been widely used for measurement of electrical impedance of biologic tissue., Numerous in-depth studies into the electrical difference between BAPs and non-BAPs have been reported by re- searchers. $^{7-9}$ Some studies have demonstrated the existence of physiologic differences between BAPs and non-BAPs. For example, BAPs have been reported to generate greater capacitance than non-BAPs. ${ }^{10-13}$ However, skin impedance measurement may cause some errors in generating characteristics of BAPs. ${ }^{14}$ Such errors may include the frequency of applied current, skin/electrode interface, contact pressure exerted by the probe, ${ }^{15}$ duration of probe application, electrode geometry, and skin condition. ${ }^{16}$

The purpose of this study is to investigate the skin measurement for modified dry electrodes on resistance and reactance at BAPs and non-BAPs with healthy volunteers. A skin electrical model was designed for fitting of BAPs impedance values using an electrical equivalent circuit.

\section{Materials and Methods}

The modified electrodes of this research locate BAPs by moving differently compared to the conventional wet

\footnotetext{
${ }^{1}$ Biomedical Information Technology Center, School of Medicine, Keimyung University, Daegu, Republic of Korea.

${ }^{2}$ Department of Information and Communication, Kyungwoon University, Kumi, Republic of Korea.

${ }^{3}$ Department of Medical Informatics, School of Medicine, Keimyung University, Republic of Korea.

${ }^{4}$ Department of Biomedical Engineering, School of Medicine, Keimyung University, Daegu, Republic of Korea.
} 
electrode $(\mathrm{Ag}-\mathrm{AgCl})$, are measurable, have good conductivity on the electrode surface, and used gold, which is a biocompatible metal. The shape of the electrode is of coaxial type, and the electrode is composed of the measuring electrode and reference electrode. The electrode is also designed to prevent leakage of current during measurement. Additionally, a pressure gauge is attached to the electrode to investigate the effect of various pressure conditions on measurements.

Ten (10) healthy male volunteers were recruited for this study, with an age range of $21-40$ years. None of the subjects had a history of physical or mental illnesses; all subjects were restricted from medication and alcohol intake for 1 week before the experiment. All participants were briefly introduced to the experiment and the concept of basic electrical principles. During testing, subjects lay supine on a standard hospital bed at constant temperature $\left(23-25^{\circ} \mathrm{C}\right)$ with the head elevated at about $40^{\circ}$ above horizontal.

Four BAPs (PC4, PC5, PC6, and PC7) located on the antecubital region were selected (Fig. 1). These BAPs were chosen because they were straight, located on flat skin surfaces, and easily accessible for electrical measurements. The locations of BAPs were identified by a qualified acupuncturist. Non-BAPs were located at $5 \mathrm{~mm}$ in the medial and parallel direction to each BAP. Only the left forearm of the subjects was used for testing. In certain cases, where scarring, cysts, or skin inflammation precluded testing on the left side, the right arm was used instead.

Most traditional methods for measurement of skin impedance were applied with amplitude of 1-25 $\mu \mathrm{A}$. However, it should be noted that the Committee on Electrocardiology recommends amplitude of $10 \mu \mathrm{A}$ or less for patientconnected leads. ${ }^{17}$ Electrical impedance of the biologic tissue

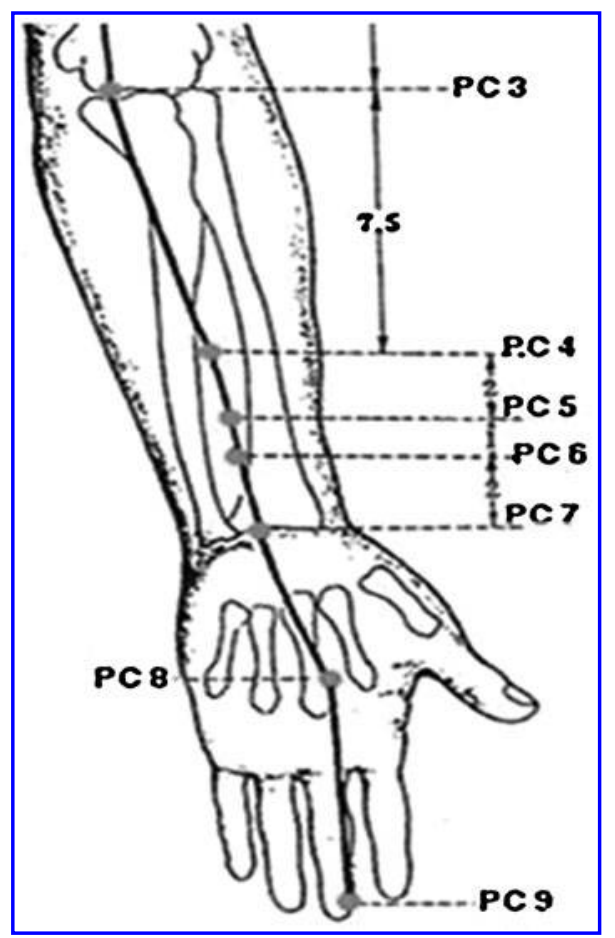

FIG. 1. The location of acupuncture points as biologic active points along the pericardium channel. was calculated from the phase difference between the electric current and the applied voltage. The skin impedances were measured in the frequency range of $1 \mathrm{~Hz}-1 \mathrm{kHz}$. This skin impedance measurement system consists of a digital lock-in amplifier (SR830, Standard Research Systems), DC voltage source (Hioki, 7016), and a Labview control system (National Instrument PXI), and is controlled by a personal computer through general purpose interface bus, as shown in Figure 2. True monopolar measurements were obtained by addition of a three-electrode system to the amplifier. The inner part of the concentric electrode was used as a measuring electrode, and the outer part as a reference electrode. The cable to the inner electrode was a coaxial type with a grounded shield. The space between these two electrodes was filled with epoxy adhesive resin. Impedance measurements with the modified dry electrode system were carried out using a lockin amplifier and voltage source connected to a concentric electrode similar to the one previously described, ${ }^{18}$ with gold as electrode material (Fig. 3). Pressure applied to the electrode was adjusted to $0.98-5.88 \mathrm{~N}$ with a coiled spring. All bio-impedance measurements and data processing were conducted automatically using a PC. The ground electrode is attached at least about $10 \mathrm{~cm}$ away from the measurement electrode. For the grounding, the return path for the current and band-type bio-impedance strip electrode (BIOPAC EL 506) was used. Pressure of the electrode on the skin was sustained as constant $(5.88 \mathrm{~N})$ during the recordings, as the electrodes were not touched during the course of the experiment. If the pressure on the skin was too large, the electrode would retract before damage occurred.

The resistance and reactance of BAPs were measured for the electrical impedance characteristics of low frequency range $(1 \mathrm{~Hz}-1 \mathrm{kHz})$. Reactance often implies polarization of stationary molecules, such as membranes and proteins, within an electrical field similar to a dielectric material embedded between capacitor plates. Inductance appears not to play a significant role in biologic systems. The relative contributions of resistance and capacitance toward the total impedance are frequency dependent.

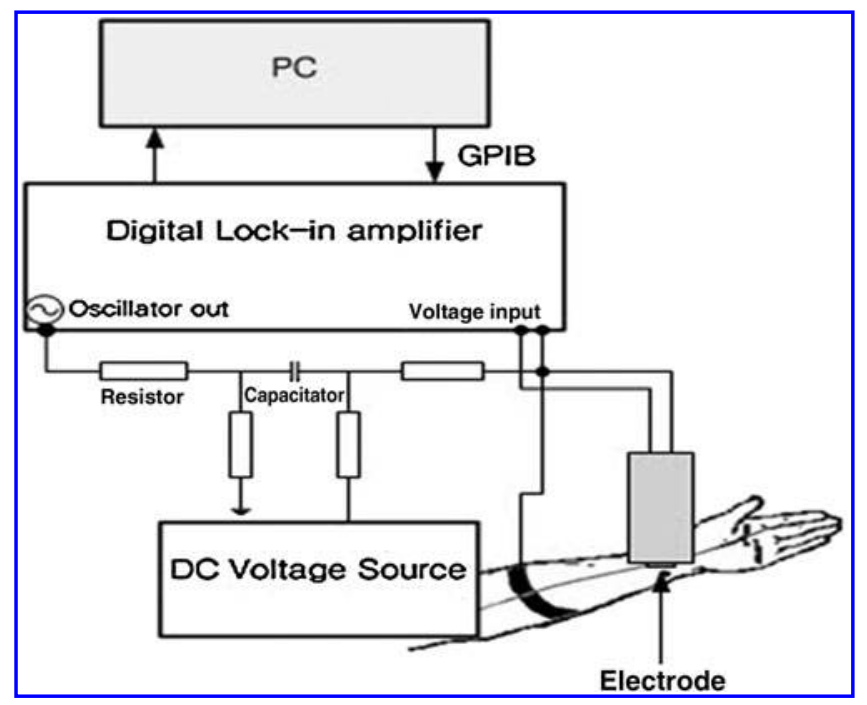

FIG. 2. Block diagram of the biologic active points measurement system. GPIB, general purpose interface bus. 


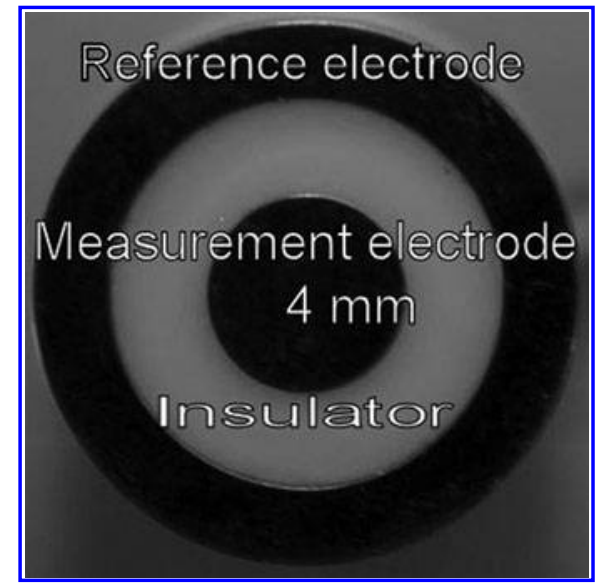

FIG. 3. Concentric electrode as constructed.

\section{Results}

BAP measurement was conducted at 2 PM daily for 1 week in order to minimize the effect of time and environment and obtain reproducible results. Measurements were taken 10 times per person and the measurement values shown are the average values. A pressure-checking test was performed in order to achieve the proper pressure of the proposed dry measuring system before it was applied for measurement of BAPs. The relationship between pressure and skin resistance are shown in Figure 4A and B. Using a dry electrode system, various contact pressures ranging from $0.98 \mathrm{~N}$ to $5.88 \mathrm{~N}$ were applied to BAPs and non-BAPs for assessment of electrical resistance. Experimentation regarding the resistance change from the pressure of pressing down, which is proposed in this study, was conducted and the results shown in Figure 4A and $\mathrm{B}$ indicate that the pressure condition of $5.88 \mathrm{~N}$ could be most reliably measured. Furthermore, the measurement value did not vary at this condition, which is expected to contribute to the reproducibility of the measured value at this condition. Dry electrode was used with no conductive gel. Impedance is decreased according to pressure due to the permeation effect of skin. Through the pressure test, the proper pressure was determined. The results of pressure tests are shown in Figure $4 \mathrm{~A}$ and $\mathrm{B}$. For dry electrodes, artifacts arise from the disturbance of the double layer region at the electrode surface. Therefore, in order to overcome the inherent problems of dry electrodes, the most reliable pressure condition of $5.88 \mathrm{~N}$ was obtained through the experimentation of this study, and so this condition was applied to subjects.

The pericardium meridian (PC) and the bladder meridian (BL) were studied in each subject. The locations of the acupuncture points and meridians were identified by an acupuncture point/meridian map of the human body in shown in Figure 1. Resistance and reactance measurements were taken at PC-4, PC-5, PC-6, and PC-7 BAPs on the left forearm. Figure $5 \mathrm{~A}-\mathrm{D}$ shows skin complex impedance value generated by the dry electrode system with the frequency range of $1 \mathrm{~Hz}-1 \mathrm{kHz}$. All four BAPs and non-BAPs were used. The resistances of four BAPs were found to decrease to about $29 \%-59 \%$ of non-BAPs and reactance of BAPs was found to decrease to about $23 \%-41 \%$ of non-BAPs. Skin impedances at BAPs were lower than those at non-BAPs.

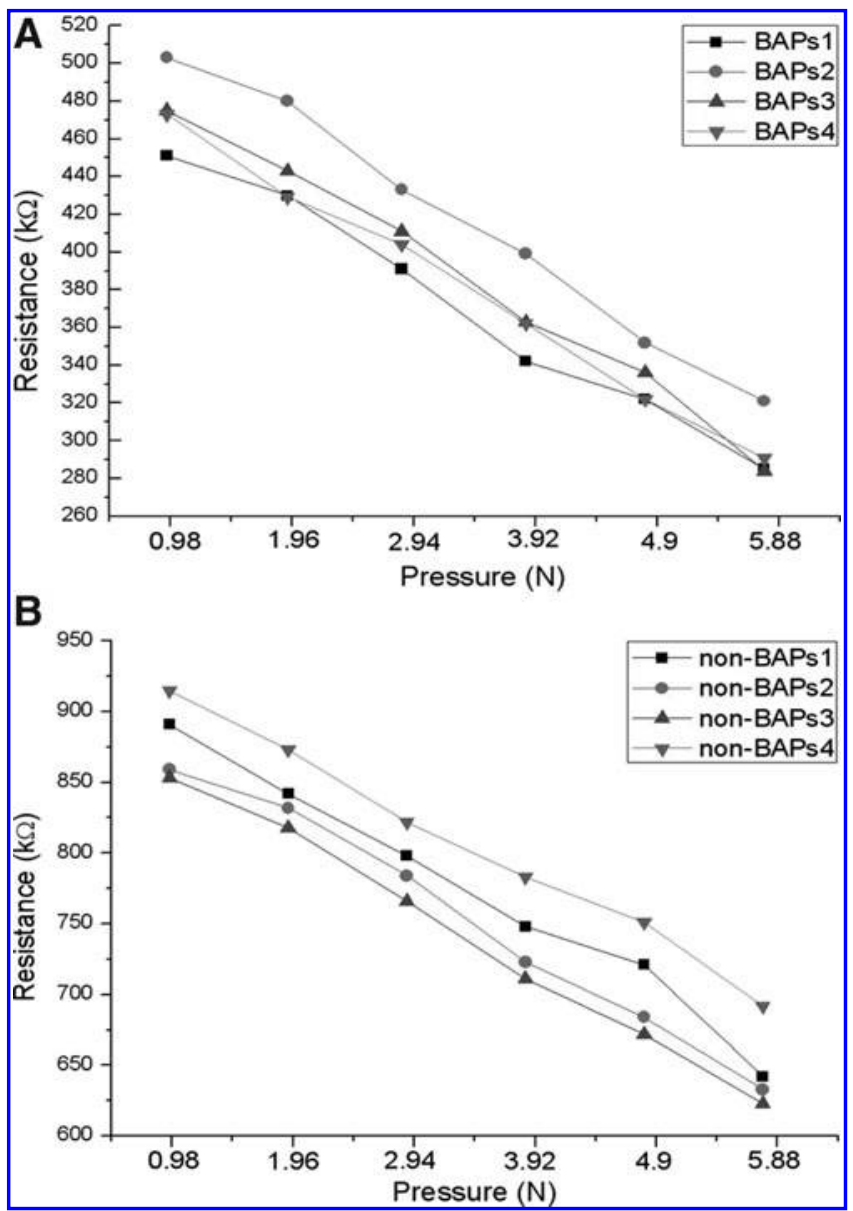

FIG. 4. Electrical resistance of biologic active points (BAPs) and non-BAPs with different contact pressures.

Figure 5A-D shows the frequency-dependent curves of skin resistance and reactance, respectively, for BAPs measurement. In addition, the resistance and reactance of BAPs skin were decreased compared with non-BAPs, and the decrease was especially marked at low frequencies $(<25 \mathrm{~Hz})$. According to this hypothesis, this study generates a backward electromotive force by polarizing a cell-wall electric potential or a defense mechanism by the body in response to an external stimulus. It also identifies electrical conduction points having greater differences than other points. When BAPs and non-BAPs are compared, current is not equal over the entire skin region, and both the electrical conduction point and the BAPs respond with lower resistance and higher capacitance. These observed differences can be used to distinguish between BAPs and non-BAPs.

Table 1 shows the result of the resistance and reactance measurements for each BAPs. In this result, the resistance and reactance of BAPs were decreased compared with non-BAPs, and the decrease was especially marked at low frequencies $(<25 \mathrm{~Hz})$. Thus, it could be possible to detect BAPs skin based on change in resistance and reactance. As shown in Figure 6, the electrical skin model originally proposed by Martinsen et al. ${ }^{19}$ was further modified and used for measurement of impedance. In this electrical model, $R_{1}$ represents the resistance of internal tissue, $R_{2}$ the resistance, and CPE1 (constant phase element) of BAPs tissues. $\mathrm{R} 3$ represents the resistance of 

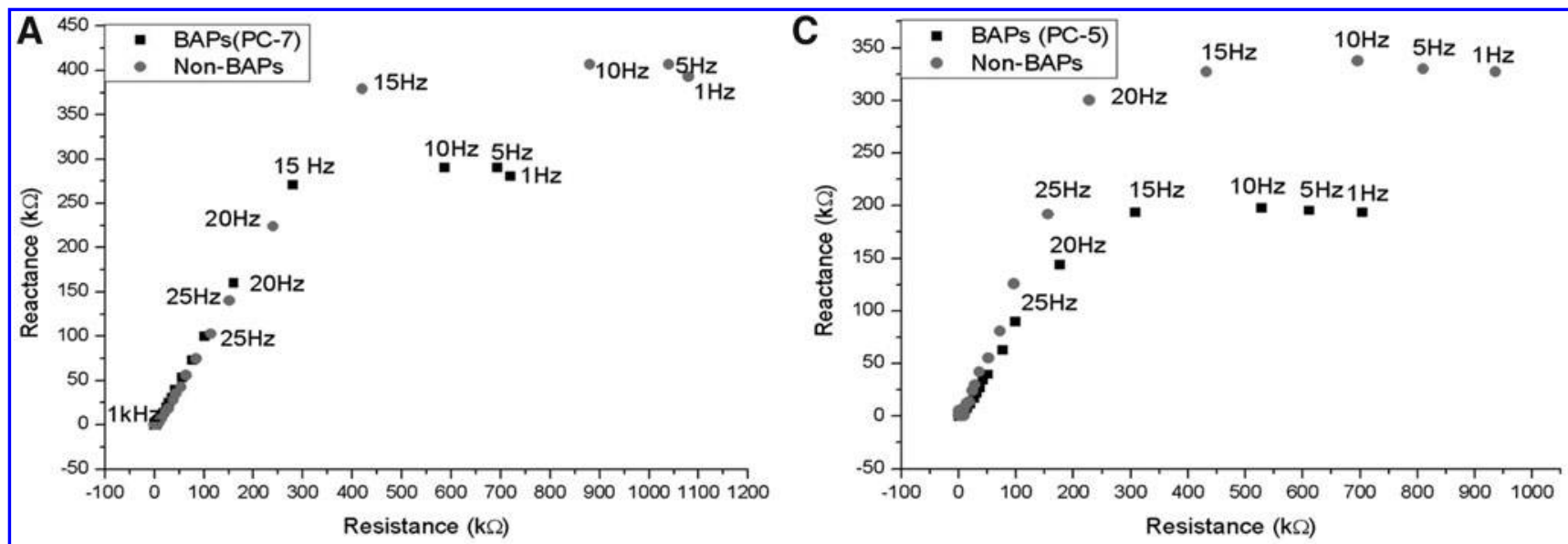

B
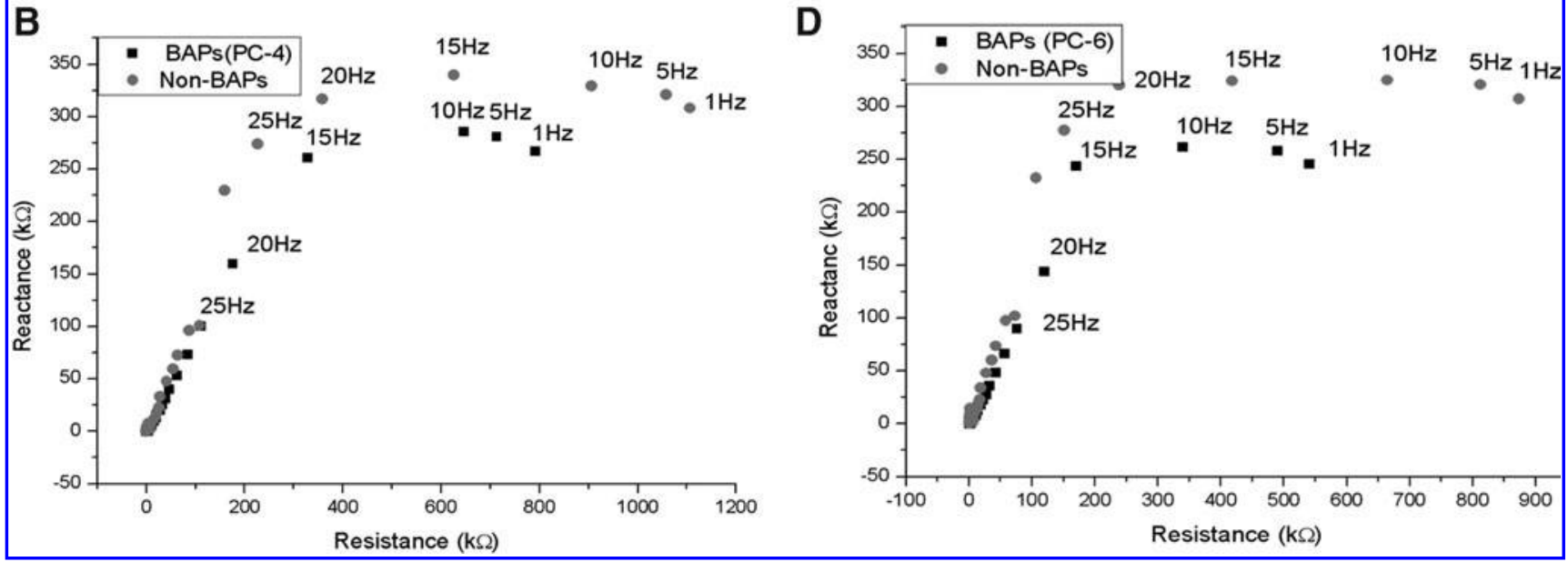

FIG. 5. Electrical impedance at PC5 and its corresponding non-biologic active points (BAPs). BAPs showed lower resistance and reactance values, particularly around the lower frequency range.

epidermis tissue, the CPE model, and C the electrode/tissue interface. This proposed electrical circuit model demonstrated the dependence of impedance on frequency with linear regression of impedance. Table 2 shows the results of components of equivalent electrical circuits that were used for skin and BAPs. From the electric circuit model, epidermis tissue has a high resistance characteristic, and R2, which represents BAPs, has low resistance. A 0.5-1 range of value was used for the parameter to represent the characteristic of the medium. The graph in Figure 7 shows results similar to the experimental results in Figure 5A-D and Table 1. Resistance and reactance of BAPs and non-BAPs were compared according to frequency. When the frequency was $15 \mathrm{~Hz}$, the ranges of re-

Table 1. Result of Resistance and Reactance Measurements for Each Biologic Active Points (BAPs)

\begin{tabular}{lcccccccc}
\hline & \multicolumn{3}{c}{ Resistance $(k \Omega)$} & & \multicolumn{3}{c}{ Reactance $(k \Omega)$} \\
\cline { 2 - 3 } \cline { 7 - 8 } Subject & BAPS & $\begin{array}{c}\text { Non- } \\
\text { BAPS }\end{array}$ & $\begin{array}{c}\text { Difference } \\
(\%)\end{array}$ & & BAPs & $\begin{array}{c}\text { Non- } \\
\text { BAPs }\end{array}$ & $\begin{array}{c}\text { Difference } \\
(\%)\end{array}$ \\
\hline 1 & 280 & 420 & 33 & & 270 & 378 & 29 \\
2 & 328 & 626 & 48 & & 260 & 339 & 23 \\
3 & 308 & 432 & 29 & & 193 & 327 & 41 \\
4 & 170 & 417 & 59 & & 244 & 324 & 25 \\
\hline
\end{tabular}

sistance and reactance of BAPs were $170(\mathrm{k} \Omega)-310(\mathrm{k} \Omega)$ and $193(\mathrm{k} \Omega)-270(\mathrm{k} \Omega)$, respectively. In the case of non-BAPs, the ranges were $417(\mathrm{k} \Omega)-626(\mathrm{k} \Omega)$ and $324(\mathrm{k} \Omega)-378(\mathrm{k} \Omega)$. From comparison of results, similar characteristics were observed between the value based on the proposed electric circuit model and the value based on simulations.

\section{Discussion}

Conventional wet electrodes systems require a long application time, and stabilization is needed for achievement of

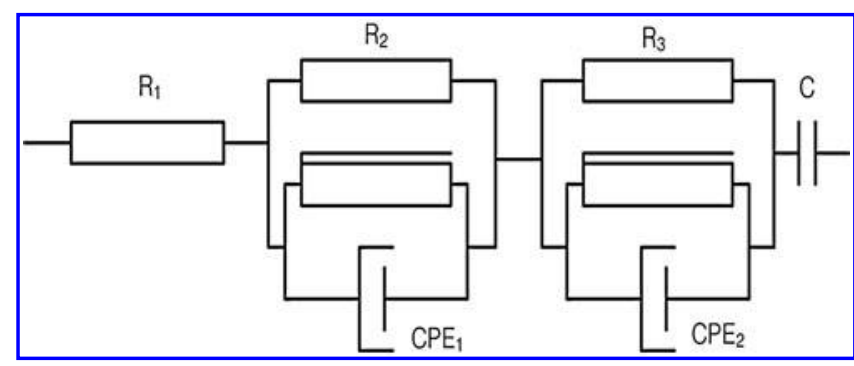

FIG. 6. The electrical equivalent circuit model of biologic active points. $\mathrm{CPE}$, constant phase element; $\mathrm{R}_{1}$, resistance of internal tissue, $R_{2}$ the resistance; $R_{3}$, resistance of epidermis tissue; $\mathrm{C}$, electrode/tissue interface. 
Table 2. Results of Components of Equivalent Electrical Circuits Used for SKin and Biologic Active Points (BAPs)

\begin{tabular}{lcrrr}
\hline Components & $R(k \Omega)$ & $\mathrm{CPE} 1(\mu \mathrm{F})$ & $\mathrm{CPE} 2(\mu \mathrm{F})$ & $\mathrm{C}(\mathrm{F})$ \\
\hline BAPs skin & $\mathrm{R} 1=1$ & $\mathrm{CPE}=0.02$ & $\mathrm{CPE}=1.1$ & $\mathrm{C}=0.001$ \\
& $\mathrm{R} 2=20$ & $\alpha=0.93$ & $\alpha=0.92$ & \\
& $\mathrm{R} 3=1100$ & & &
\end{tabular}

$\mathrm{R}$, resistance; $\mathrm{CPE}$, constant phase element; $\mathrm{C}$, electrode/tissue interface.

sufficient diffusion of the electrolytic gel into the skin. Furthermore, gel will dry after a few hours and can cause skin irritation. Performance with respect to power-line interference of the wet type is limited by electrode/skin contact impedance. Artifact from modified electrode also reduces over time because the electrodes and their housing will tend to adhere to the skin as perspiration accumulates, reducing the relative movement between skin and electrode surfaces in the local area. To take readings, pressure has to be applied to the modified electrode in order to get a good contact between the electrodes and the skin. The new measurement method using modified dry electrodes may mitigate the disadvantages of wet electrodes, including long preparation time before measurement and difficulty of measurement, making simple and efficient measurement possible.

Without knowledge of the exact sizes of the BAPs tested in this study, it can only be said that the 4-mm-diameter area centered over what was defined by the acupuncturist as a BAPs had lower, higher, or the same resistance as a 4-mmdiameter area a few centimeters away from that of presumed BAPs. The authors believe that a better way to test the hypothesis that BAPs have lower resistance than non-BAPs would be to measure the skin immediately surrounding the approximately 4-mm area defined as the BAPs and then determine whether a given BAPs has relatively lower resistance than that of the immediately adjacent skin. According to this system at four BAPs and their corresponding nonBAPs sites, it is suggested that differences in skin impedance between BAPs and non-BAPs sites exist, where this system will be capable of distinguishing them.

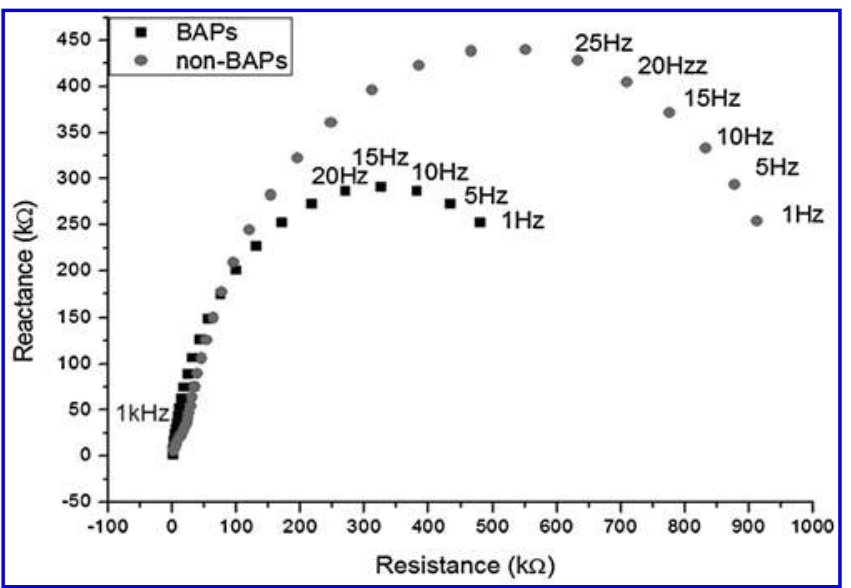

FIG. 7. Result of biologic active points (BAPs) and nonBAPs using an electrical equivalent model.
In the present measurement system, it was possible to distinguish the characteristics between BAPs and non-BAPs. Thus, it is predicted that objective and scientific analyses using electrical methods would be possible.

In the bio-impedance electrical model, the CPE together with a circular arc complex impedance analysis were introduced by Cole as early as $1923 .^{20}$ The impedance model was developed gradually in the 1930s until its completion with the mathematical equation. In 1941, Cole introduced a similar model for dielectric permittivity, the Cole-Cole model. ${ }^{21}$ The electrical impedance model used in this study includes a CPE. This model is in general better suited for tissue and cell suspensions, and has often been used implicitly.

\section{Conclusions}

The modified electrode measurement system is clarified for the electrical impedance characteristics of low frequency range $(1 \mathrm{~Hz}-1 \mathrm{kHz})$. The measurement impedance values are found to be alike from our proposed electrical circuit model. This will be helpful in scientific analysis and verification of the electric characteristics of BAPs. In general, BAP measurement systems have been problematic due to a deficiency of reproducible measurements. However, the present measurement system is stable and is capable of automatic data measurement. With this study, it was possible to distinguish BAPs from surrounding skin by the skin impedance measurement technique with a searching electrode.

Future studies should be conducted for development of electrode systems that can accurately locate BAPs in order to unveil unknown attributes.

\section{Acknowledgment}

We would like to thank Prof. Gi Wang Kim at School of Korean Medicine of Pusan National University, Yangsan-Si, Republic of Korea for his helpful discussion and suggestions.

\section{Disclosure Statement}

No financial conflicts exist.

\section{References}

1. Chiappelli F, Prolo P, Cajulis OS. Evidence-based research in complementary and alternative medicine I: history. Evid Based Complement Alternat Med 2005;2:453-458.

2. Chen KG. Electrical properties of meridians. IEEE Med Biol 1996;15:58-63.

3. Comunetti A, Laage S, Schiessl N, Kistler A. Characterisation of human skin conductance at acupuncture points. Experentia 1995;51:328-331.

4. Ahn AC, Martinsen OG. Electrical characterization of acupuncture points. J Altern Complement Med 2007;13:817-824.

5. Yu Jeong Choi, June Soo Kim et al. The change of pacing lead impedance over time: Difference in the atrial and ventricular lead. Korean Circulation J 2005;35:454-459.

6. Byung Ki Lee, Joo Sang Park et al. Effect of thoracentesis on thoracic impedance and cardiac performance. Korean Circulation J 1984;14:17-22.

7. Kwok G, Cohen M, Cosic I. Mapping acupuncture points using multi channel device. Australas Phys Eng Sci Med 1998;21:68-72.

8. Halek J, Opavsky J, Kolarova J. Problems of the skin resistance measuring in randomly chosen and so-called active 
points of the skin. Acta Univ Palacki Olomuc Fac Med 1984;107:51-62.

9. Cho SH, Chun SI. The based electrical skin resistance of acupuncture points in normal subjects. Yonsei Med J 1994;35:464-474.

10. Johng HM, Cho JH, Shin HS, et al. Frequency dependence of impedances at the acupuncture points Quze (PC3). IEEE Eng Med Biol Mag 2002;21:33-36.

11. Barlea NM, Sibianu H, Ciupa RV. Electrical detection of acupuncture points. Acta Electrotech Napocensis 2000;41:59-61.

12. Prokhorov EF, González-Hernandez J, Vorobiev YV, et al. The electrical characteristics of human skin and biological active points in vivo. Med Biol Eng Comput 2000;38:507-511.

13. Zhang $\mathrm{W}, \mathrm{Xu} \mathrm{R}, \mathrm{Zhu} \mathrm{Z}$. The influence of acupuncture on the impedance measured by four electrodes on meridians. Acupunct Electrother Res 1999;24:181-188.

14. Martinsen OG, Grimnes S, Mørkrid L, Hareide M. Line patterns in the mosaic electrical properties of human skin. IEEE Trans Biomed Eng 2001;48:731-734.

15. Fang O, Dedi R, Ahmed B, Cosic I. Comparison of Skin Resistance Between Biological Active Points of Left and Right hands with various Contact Pressures. In: Proceedings of the 26th Annual International Conference of the IEEE EMBS San Francisco, CA, USA. 2004:1-5.

16. Pearson S, Colbert AP, McNames J, et al. Electrical skin impedance at acupuncture points. J Altern Complement Med 2007;13:409-418.
17. Laks M, Arzbaecher R, Bailey J, et al. Recommendations for safe current limits for electrocardiographs. Circulation 1996; 93:837-839.

18. Yamamoto Y, Yamamoto T, Ozawa T. Characteristics of skin admittance for dry electrodes and the measurements of skin moisturization. Med Biol Eng Comput 1986;24:71-77.

19. Martinsen OG, Grimnes S, Karlsen J. Electrical methods for skin moisture assessment. Skin Pharmacol 1995;8: 237-245.

20. Cole KS. Electrical impedance of suspensions of spheres. J Gen Physiol 1928;12:29-36.

21. Cole K, Cole S. Dispersion and absorption in dielectrics. J Chem Phys 1941;9:341-351.

Address correspondence to: Yoon-Nyun Kim, MD, PhD Biomedical Information Technology Center School of Medicine Keimyung University 2800 Dalgubeoldaero, Dalseo-gu Daegu 704-701 Republic of Korea

E-mail: ynkim@dsmc.or.kr 